\title{
CRT contraindicated in patients with short QRS duration
}

The EchoCRT trial has shown that cardiac resynchronization therapy (CRT) is not beneficial and can cause harm in patients with heart failure (HF) and a QRS duration $<130 \mathrm{~ms}$. The findings of this landmark international study, in which an expanded indication for CRT was tested, were presented at the ESC Congress in September 2013 and published in NEJM.

CRT is a guideline-recommended treatment strategy for patients with moderate-to-severe HF, left ventricular ejection fraction (LVEF) $<35 \%$, and a QRS

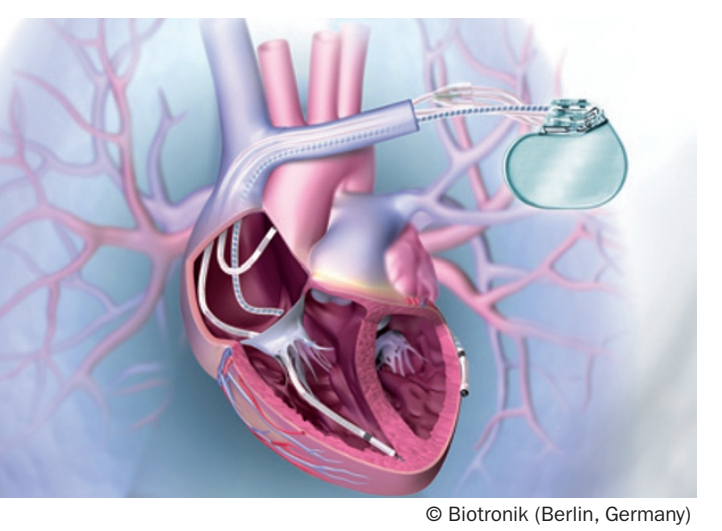

duration $\geq 120 \mathrm{~ms}$. Some evidence exists from small studies that CRT might also benefit individuals with a narrow QRS complex, if they exhibit left ventricular dyssynchrony. "The EchoCRT trial evaluated an important question for daily practice," commented investigator Johannes Holzmeister. "The results will help to guide physicians' treatment decisions for [patients with] HF."

Trial participants with NYHA class III HF, LVEF $\leq 35 \%$, QRS $<130 \mathrm{~ms}$, and left ventricular dyssynchrony were enrolled over a $~ 5$-year period at 115 centres. All patients were implanted with a Biotronik (Berlin, Germany) Lumax HF-T device. Patients were randomly assigned to have the CRT function switched on ( $n=404$; CRT group) or off ( $n=405$; control group).

During follow-up (mean 19.4 months), the incidence of the primary outcome (allcause death or hospitalization for heart failure) did not differ significantly between the two groups (CRT 28.7\% vs control $25.2 \%)$. However, more patients in the CRT group than in the control group died
(11.1\% vs 6.4\%; HR 1.81, 95\% CI 1.11-2.93, $P=0.02)$, primarily from cardiovascular causes $(9.2 \%$ vs $4.2 \% ; P=0.004)$. Although "a nominally significant increase in mortality in patients receiving CRT was observed ... these data have to be interpreted with great caution, since the trial was stopped prematurely for futility and the vital status of a number of subjects could not be confirmed at the end of the study," explained Dr Holzmeister Also of concern in the CRT group were the significantly increased rates of serious device-related adverse events, and of inappropriate cardioverter-defibrillator shocks.

In an editorial that accompanied the trial report, Clyde Yancy and John McMurray conclude that "the simple 12-lead surface ECG remains the only evidence-based means of identifying patients who may obtain the substantial benefits of CRT."

\section{Alexandra Roberts}

Original article Ruschitzka, F. et al. Cardiac-resynchronization therapy in heart failure with a narrow QRS complex. N. Engl. J. Med. doi:10.1056/NEJMoa1306687 